\title{
Real-Time Monitoring of Nanoscale Polarization Switching
}

\author{
Researchers have visualized the nanoscale jumps in a ferroelectric's \\ polarization that are thought to play a key role in how well some \\ ferroelectric devices function.
}

By Rachel Berkowitz

E lectrically polarize a ferroelectric material, and tiny, discontinuous jumps can appear in the strength of its polarization. These jumps, known as Barkhausen pulses, create noise that can inhibit the functionality of nanoscale optical and electronic devices that rely on polarization switching. As such, researchers want to understand the pulses' origin. Until now, however, the pulses had not been individually observed. Now, using a technique that they developed for imaging the real-time motion of ferroelectric domains, Vasiliki Tileli and her colleagues at the Swiss Federal Institute of Technology in Lausanne have observed Barkhausen pulses at the nanoscale [1]. The researchers hope that their demonstration will lead to a better understanding of the kinetics of polarization switching, enabling its use in novel devices for electro-optical, computing, and data-storage applications.
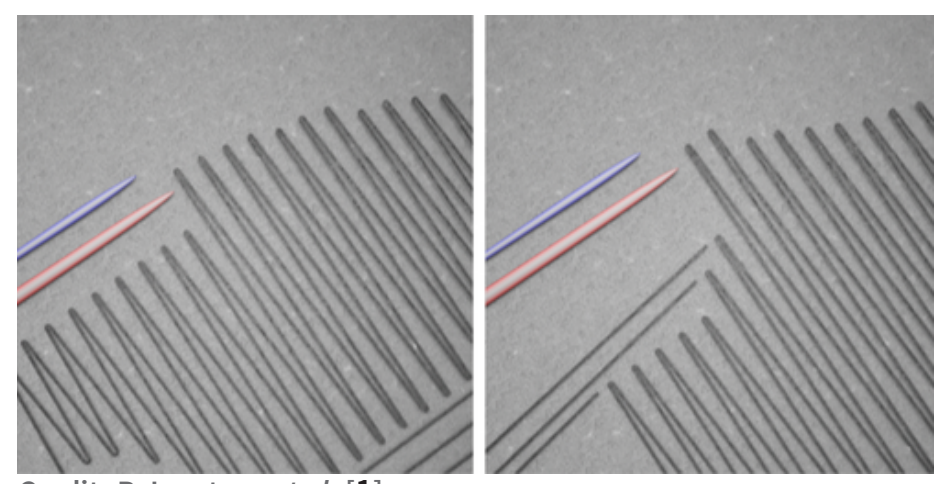

For their experiments, the researchers placed a thin layer of a $\mathrm{BaTiO}_{3}$, a single-crystal ferroelectric, on a microdevice.

$\mathrm{BaTiO}_{3}$ consists of groups of 33-nm-wide needle-shaped domains. Using a transmission electron microscope, they tracked the motion of the needles when they applied an electric field to the $\mathrm{BaTiO}_{3}$.

The needles formed a herringbone pattern and could move forward and backward upon application of the electric field. Observations of the needles' motions showed that when an individual needle jumped forward, the one perpendicular to it retracted. Tileli and her colleagues conclude that these discrete jumps are individual Barkhausen pulses. They think that they arise from extended electromechanical fields around the needle tips that pin their movement until a critical activation energy causes them to move.

Rachel Berkowitz is a Corresponding Editor for Physics based in Vancouver, Canada.

\section{REFERENCES}

1. R. Ignatans et al., "Individual Barkhausen pulses of ferroelastic nanodomains," Phys. Rev. Lett. 127, 167601 (2021).

Credit: R. Ignatans et al. [1] 